\title{
thebmj
}

Practice Quality Improvement Report

\section{Comprehensive care and HIV prophylaxis after sexual assault in rural South Africa: the Refentse intervention study}

BMJ 2009; 338 doi: http://dx.doi.org/10.1136/bmj.b515 (Published 13 March 2009) Cite this as: BMJ 2009;338:b515

Julia C Kim, senior researcher ${ }^{1}$, clinical research fellow2, lan Askew, director ${ }^{3}$, Lufuno Muvhango, sexual violence programme coordinator ${ }^{1}$, Ntabozuko Dwane, MMed research intern 1, Tanya Abramsky, research fellow in epidemiology2, Stephen Jan, senior health economist 4 , Ennica Ntlemo, research nurse 1 , Jane Chege, programme associate 5 , Charlotte Watts, Sigrid Rausing professor 2

${ }^{1}$ RADAR, School of Public Health, University of the Witwatersrand, Acornhoek, South Africa

${ }^{2}$ Gender, Violence and Health Centre, Health Policy Unit, Department of Public Health and Policy, London School of Hygiene and Tropical Medicine, London WC1E 7HT

${ }^{3}$ FRONTIERS in Reproductive Health Program, Population Council, Nairobi, Kenya

${ }^{4}$ The George Institute for International Health, Sydney, Australia

${ }^{5}$ FRONTIERS in Reproductive Health Program, Southern Africa, Population Council, Johannesburg, South Africa

Correspondence to: J Kim jkim@agincourt.co.za

- Accepted 26 August 2008

\section{Abstract}

Problem Although international guidelines specify the central role of the health sector in providing comprehensive care, including HIV post-exposure prophylaxis (PEP), after sexual assault, in both industrialised and developing countries there are many challenges to providing timely and comprehensive services.

Design A nurse driven model of post-rape care was integrated into existing hospital services; the before and after study design evaluated impacts on quality of care, reviewing 334 hospital charts and conducting interviews with 16 service providers and 109 patients.

Setting 450 bed district hospital in rural South Africa.

Key measures for improvement Quality of care after rape (forensic history and examination, provision of emergency contraception, prophylaxis for sexually transmitted infections, referrals); provision of HIV counselling and testing and provision and completion of full 28 day course of PEP; and service utilisation (number of service providers seen on first visit and number of rape cases presenting to hospital per month). 
Strategies for change After completing baseline research, we introduced a five part intervention model, consisting of a sexual violence advisory committee, hospital rape management policy, training workshop for service providers, designated examining room, and community awareness campaigns.

Effect of change Existing services were fragmented and of poor quality. After the intervention, there were considerable improvements in clinical history and examination, pregnancy testing, emergency contraception, prophylaxis for sexually transmitted infections; HIV counselling and testing, PEP, trauma counselling, and referrals. Completion of the 28 day course of PEP drugs increased from $20 \%$ to $58 \%$.

Lessons learnt It is possible to improve the quality of care after sexual assault, including HIV prophylaxis, within a rural South African hospital at modest cost, using existing staff. With additional training, nurses can become the primary providers of this care.

\section{Introduction}

Sexual violence can have a profound impact on the physical, psychological, and social wellbeing of rape survivors. In addition to immediate genital and bodily injuries, risks include HIV and other sexually transmitted infections, unwanted pregnancy, urinary tract infections, chronic pelvic pain, miscarriage, depression, substance abuse, post-traumatic stress disorder, and suicide.1 International guidelines specify the central role of the health sector in providing comprehensive clinical care after sexual assault.2 The recent emergence of HIV post-exposure prophylaxis (PEP)—a course of antiretroviral drugs - to reduce risk of HIV transmission after sexual assault has heightened the importance of efficient service delivery. 3 International guidelines recommend giving PEP within 72 hours, 24 and even in countries where it is not currently available, other important aspects of care after sexual assault are time dependent, including emergency contraception and collection of forensic evidence.

The range of skills and services that must be coordinated to deliver expedient, effective care to rape survivors needs an integrated, multisectoral delivery model. This can prove enormously challenging to health systems, particularly in under-resourced settings.5 6 To date, few health sector interventions for sexual assault have been rigorously evaluated.7 Between March 2003 and August 2006 we conducted the Refentse study (meaning "we shall overcome" in Venda) to evaluate a nurse driven, comprehensive, model for care after rape that was integrated into existing services in a rural South African hospital. The 450 bed district hospital functioned as a referral centre for surrounding clinics, and rape cases were treated within the hospital's outpatient department, which is staffed by a team of six doctors and 10 nurses on average.

\section{Outline of problem}

We used formative research to conduct a baseline assessment of care after sexual assault and to design the intervention model, which consisted of a sexual violence advisory committee, a hospital rape management policy, training workshops for service providers, centralisation and coordination of care through a designated examining room, and community awareness campaigns. 8 The key problems identified at baseline were absence of institutional policies or treatment protocols for care after sexual assault; fragmented service delivery resulting in multiple interactions with service providers and subsequent delays; lack of relevant training and negative attitudes of service providers; substandard provision of clinical care; lack of privacy; poor collection of forensic evidence; and lack of counselling about drug treatment, trauma counselling, and psychosocial referral. Obstacles to timely provision of voluntary HIV counselling and testing and about PEP were largely due to institutional and provider 
barriers, rather than patients' delays in seeking care.9 Given the shortage of doctors (common in many settings), a key question for the research was whether the service could be primarily nurse driven.5

\section{Key measures for improvement}

We used a before and after design to assess whether the intervention led to improvements in the quality of care after rape (forensic history and examination, provision of emergency contraception, prophylaxis for sexually transmitted infections, and referrals); the provision of post-exposure prophylaxis (HIV counselling and testing, and provision and completion of a full 28 day regimen of zidovudine and lamivudine, as recommended by South African guidelines); and the efficiency and utilisation of the service (number of service providers seen on first visit and number of rape cases presenting to hospital each month).

\section{Process of gathering information}

To document the quality of care at baseline and to measure change after the intervention we used both qualitative and quantitative methods. Data were extracted from medical charts by two doctors external to the hospital and intervention.

\section{Facility inventories}

We used national and international guidelines to develop a checklist of tools and resources available in hospitals and health centres. 210 To document the availability of relevant tools and resources, we conducted an inventory of the hospital at baseline and again 10 months after introducing the intervention.

\section{Reviews of hospital charts}

Measuring against standards set by the National Department of Health,10 a structured chart review form assessed quality of clinical care, including thoroughness of history and forensic examination, laboratory investigations, drugs, and referrals. To minimise observer bias, specific criteria were established for assigning quantitative values to each indicator. For example, to assess quality of forensic examination, a scoring system was developed to document whether healthcare workers had made note of a range of factors (for example, evidence of injury) required in the sexual assault evidence collection kit. Criteria were established to determine whether patients had been eligible for, and received, relevant diagnostic tests and treatment. All cases of rape presenting to the outpatients department and recorded in its rape register between 1 March 2003 and 31 August 2006 (42 months) were eligible for review, regardless of age, sex, or time of presentation. If a chart could not be retrieved from medical records at first request, a second attempt was made; failing this, the record was excluded from the study (fig 1) $\underline{\Downarrow}$.

Fig 1 Enrolment in the study

\section{Key informant interviews with service providers}

Semistructured interviews were conducted with 16 service providers (doctors, nurses, social workers, a pharmacist, and police officers) involved in post-rape care. Questions explored common attitudes, practices, and perceived obstacles to service delivery before and after the training. 


\section{Interviews with patients}

A structured interview was used to document patients' experiences of care, including completion of the course of drugs, uptake of referrals, and counselling, as well as more subjective impressions of quality of care. Completion of the course of drugs was assessed by using a calendar and visual recognition tool, which contained unlabelled samples of each drug. Respondents were asked a series of questions: Was this medication given to you? If so, what do you think it was for? How did you take it (dose, interval, and duration)? How many pills remained when you stopped?

All cases of rape recorded in the outpatient department's rape register between 20 June 2004 and 31 August 2006 (26 months, beginning after formative research) were eligible for interview. At the end of their visit to the outpatient department, patients were informed about the study and offered a follow-up appointment in four weeks. Informed consent was sought to contact the patient or their guardian by telephone one week before the return appointment, to remind them of the interview. Because formative research indicated that return rates would be low because of economic constraints, patients were offered a travel stipend, to be paid on return to the hospital.

Face to face interviews were conducted in the local language in a private room by a trained female interviewer. Where patients were aged under 14, interviews were conducted with the parent or guardian. The median time between presentation to hospital and interview was 34 days. Data collection was informed by international guidelines on technical, ethical, and safety considerations in conducting research on gender based violence.11 12

\section{Analysis and interpretation}

\section{Chart reviews}

Among the 409 cases recorded in the rape register during the study period, $334(82 \%)$ medical charts were successfully reviewed. Cases assessed before and after the intervention were similar in terms of patients' age, sex, time of presentation, proportion opening a police report, and eligibility criteria for diagnostic tests and treatment.9 Both before and after the intervention, documented cases were almost exclusively female, with mean age of 20 years (range 3 months to 94 years), and more than one quarter were children under 14. Most presented within 72 hours of assault and were eligible for both emergency contraception and HIV prophylaxis. Most were attended by a junior doctor rather than a senior doctor. 9

\section{Patient interviews}

Among the 330 cases recorded in the register during the interview period, 195 (59\%) patients were enrolled into the study and 109 (56\%) were successfully interviewed (fig 11). The 195 patients from whom informed consent was sought had similar characteristics to the 109 who were successfully interviewed. Those interviewed were also similar to the 334 whose medical charts were reviewed.9

Data were analysed using STATA 9.0. We compared outcomes before and after the intervention model was introduced. Risk ratios adjusting for potential confounders were estimated using Poisson regression with robust standard errors, to quantify and confirm observed patterns of intervention effect (full analysis presented elsewhere9).

\section{Strategy for change}

On the basis of the findings of the formative research, we introduced a five part intervention model in March 2005, consisting of a sexual violence advisory committee, hospital rape management policy, 
training workshop for service providers, centralisation and coordination of care through a designated examining room, and community awareness campaigns (box 1). The intervention model relied entirely on existing hospital staff, with the exception of a research nurse who provided in-service training to new nurses joining the outpatient department after the initial workshop, and the coordinator of the sexual violence advisory committee, who convened quarterly meetings of the committee and liaised with members between meetings.

\section{Box 1: Refentse intervention model for strengthening sexual assault care}

\section{Sexual violence advisory committee}

To encourage buy-in and support from relevant stakeholders, a multisectoral sexual violence advisory committee was established, and included the hospital's chief executive officer, pharmacists, psychiatric nurse, HIV clinical nurses, doctors, nursing managers in the outpatient department, social workers, and representatives from the local police station. The committee reviewed findings from the diagnostic phase in order to contribute to the design of the rest of the intervention model.

\section{Hospital rape management policy}

The sexual violence advisory committee drafted a rape management policy, which subsequently became an official hospital policy, signed by the chief executive officer and senior managers. It was based on the national management guidelines for sexual assault and laid out treatment protocols that addressed specific problems identified during the diagnostic phase: 24 hour access to counselling about HIV testing, introduction of an immediate dose of PEP drugs, and dispensing a full 28 day course of PEP on the initial visit.

\section{Training workshop for healthcare workers and other providers}

A two day training workshop for healthcare workers and other service providers was developed and implemented in March 2005. Participants were proposed by the sexual violence advisory committee and comprised senior managers and healthcare workers from the hospital, a hospital pharmacist, district representatives from the Department of Health and Social Welfare, social workers, police, and a representative from the local prosecutors office. Drawing on training expertise from the Western Cape Department of Maternal, Child, and Women's Health, the workshop focused on developing a multisectoral approach to rape management, based on key competencies outlined in the national management guidelines. Key issues were those of addressing common myths and attitudes about rape, an overview of clinical care and PEP, exploring an expanded role for nurses (including forensic examination), and strengthening relationships between the health sector, social workers, police, and local prosecutors.

\section{Centralisation and coordination of care through a designated examining room}

After the training workshop, police decided to prioritise PEP by bringing patients to hospital immediately, and opening a file on the case only after the patient had received clinical care. Hospital clerks were instructed not to redirect patients to the police station to open a case until they had been treated. Senior nursing managers decided to centralise post-rape care through a 
designated room in the outpatient department and to prioritise rape cases so they could bypass the queue in the department. With minor changes, the room was made more private, with all post-rape treatment (drugs for sexually transmitted infection, pregnancy tests, emergency contraception, HIV tests, and PEP drugs) being stocked and dispensed from a locked cupboard in the room. A set of clinical tools designed to help health care workers to implement the national management guidelines for sexual assault were made available in the room.

\section{Community awareness campaigns}

To promote community awareness about sexual violence, PEP, and availability of the post-rape services at the hospital and police station, the research nurse worked with local stakeholders (Department of Health and Social Welfare, police station, local schools, and radio station) to distribute information pamphlets and convey key messages at community HIV awareness events. In addition, nurses at local primary health care clinics were trained to include information regarding sexual violence, its effects on health, and the services available during their routine "morning health talks" for patients waiting in the queues for the clinic.

\section{Effects of change}

\section{Changes in quality of post-rape care}

After adjustment for the potentially confounding factors noted above, the quality of post-rape care improved significantly across 11 indicators (table 1$) \underline{\Downarrow}$. Provision of counselling and testing for HIV increased from $60 \%$ to $87 \%$, and drugs given for preventing sexually transmitted infections increased from $88 \%$ to $92 \%$. After the intervention patients were much more likely to have received HIV prophylaxis (whether a starter pack or full 28 day course) and to have received the full course on their first visit. Considerable improvements were also seen in prescription of anti-emetics and referrals to psychosocial services.

\begin{tabular}{|c|c|c|}
\hline Quality of care indicator & $\begin{array}{c}\text { Before } \\
\text { intervention } \\
(n=161)\end{array}$ & $\begin{array}{c}\text { After } \\
\text { intervention } \\
(n=173)\end{array}$ \\
\hline \multicolumn{3}{|l|}{ History and examination: } \\
\hline History score $>5 / 10$ & $55 / 158(35)$ & $101 / 173(58)$ \\
\hline Examination score $>3 / 5$ & $28 / 112(25)$ & $51 / 171(30)$ \\
\hline \multicolumn{3}{|l|}{ Pregnancy prevention: } \\
\hline Pregnancy test given & $52 / 77(68)$ & $86 / 100(86)$ \\
\hline Emergency contraception given & $71 / 109(65)$ & $87 / 120(73)$ \\
\hline Sexually transmitted infections: & & \\
\hline
\end{tabular}


Voluntary HIV counselling and testing and post-exposure prophylaxis (PEP):

\begin{tabular}{|c|c|c|}
\hline Any HIV counselling and testing & $89 / 148(60)$ & $113 / 130(87)$ \\
\hline HIV counselling and testing on first visit & $35 / 85(41)$ & $66 / 109(61)$ \\
\hline Any PEP drugs given & $77 / 161(48)$ & $118 / 165(72)$ \\
\hline 28 day drug course given at first visit & $11 / 73(15)$ & $59 / 107(55)$ \\
\hline Anti-emetics given & $23 / 77(30)$ & $52 / 115(45)$ \\
\hline Referral to social worker or psychiatric nurse & $30 / 161(19)$ & $79 / 173(46)$ \\
\hline
\end{tabular}

\section{Table 1}

Chart review: changes in quality of care after sexual assault. Values are numbers (percentages) of charts with information

\section{Completion of PEP}

After the intervention, patients were more likely to recognise the PEP pills and to know their purpose (table 2) $\underline{\Downarrow}$. They were more likely to report having received a full 28 day course of treatment on their first visit, and more than three times as likely to report that they had completed the full course. After training, police prioritised PEP by bringing patients to hospital before opening a police report. The hospital's rape management policy also introduced a "stat" (single immediate) dose of PEP at the start of the treatment protocol. These steps reduced the mean time to receiving the first dose of PEP from 28 hours to 18 hours after assault. This is an important outcome, as many animal studies show that prophylaxis is more efficacious the sooner it is taken, and some guidelines encourage provision within 24 hours of exposure.2 41314

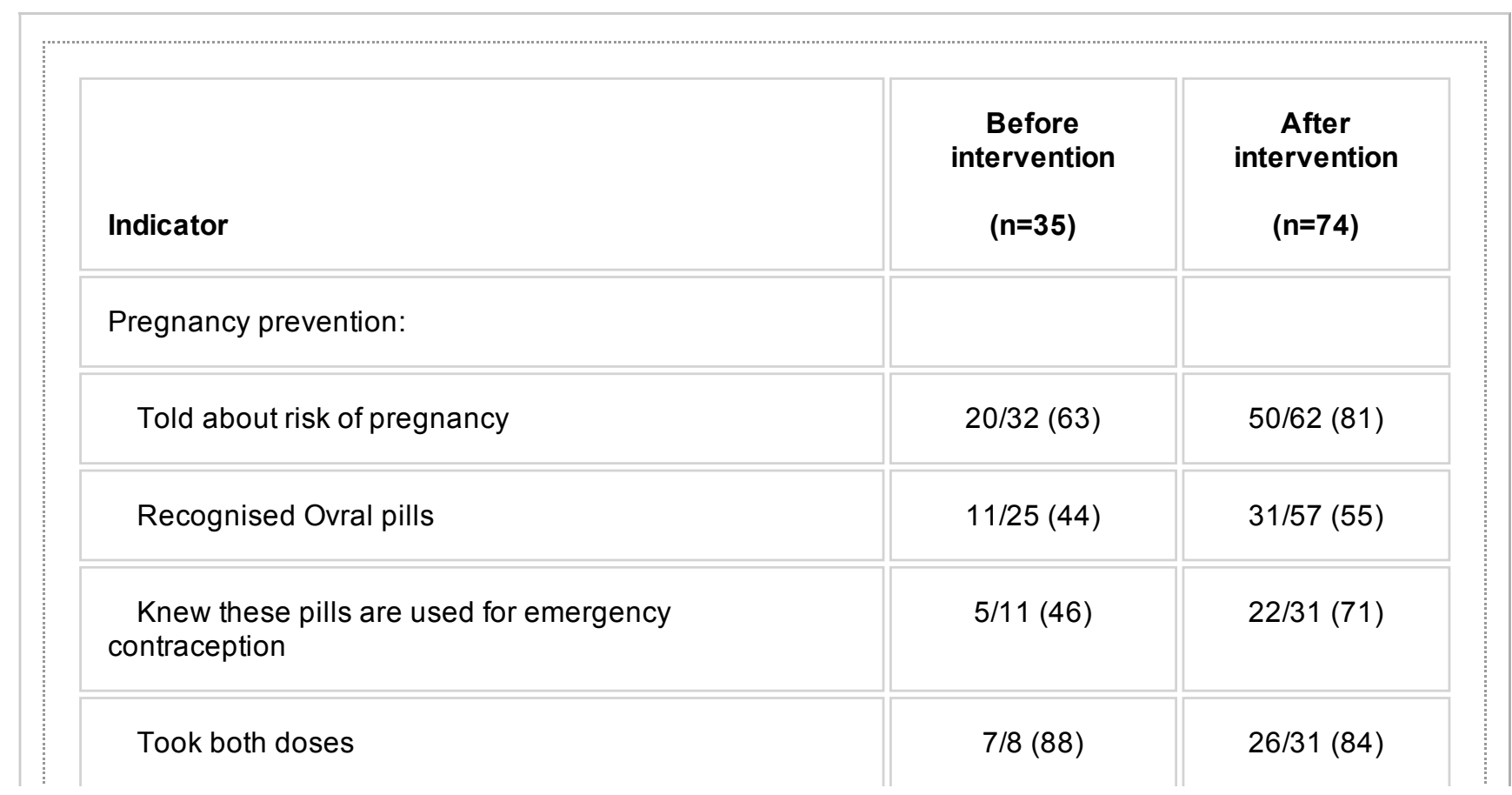


Prevention of sexually transmitted infections:

\begin{tabular}{|c|c|c|}
\hline Recognised doxycycline pills & $18 / 25(72)$ & $37 / 57(65)$ \\
\hline $\begin{array}{l}\text { Knew these pills are used to treat sexually transmitted } \\
\text { infection }\end{array}$ & $1 / 18(6)$ & $7 / 37(19)$ \\
\hline Knew correct dosing & $9 / 18(50)$ & $25 / 37(68)$ \\
\hline Took doxycycline for seven days & $4 / 14(29)$ & $12 / 29(41)$ \\
\hline \multicolumn{3}{|l|}{ Post-exposure prophylaxis (PEP): } \\
\hline Recognised PEP pills & 18/33 (55) & $51 / 68(75)$ \\
\hline Knew purpose of pills & $2 / 16(13)$ & $24 / 49(49)$ \\
\hline Knew correct dosing & $10 / 15(67)$ & $43 / 50(86)$ \\
\hline Full course of drugs given at first visit & $14 / 36(39)$ & $15 / 26(58)$ \\
\hline Took drugs for 28 days & $3 / 15(20)$ & $21 / 36(58)$ \\
\hline Had side effects of PEP drugs & $8 / 17(47)$ & $24 / 57(42)$ \\
\hline Mean (SD) hours between assault and first dose & $27.9(33.1)$ & $18.1(14.4)$ \\
\hline \multicolumn{3}{|l|}{ Efficiency of service: } \\
\hline Redirected from hospital to police & $7 / 16(44)$ & $6 / 35(17)$ \\
\hline Saw six or more care providers & $30 / 35(86)$ & $40 / 74(54)$ \\
\hline Spent four or more hours at hospital & $14 / 35(40)$ & $36 / 74(49)$ \\
\hline Received psychosocial referral & $7 / 34(21)$ & $36 / 73(49)$ \\
\hline \multicolumn{3}{|l|}{ Perceptions of hospital: } \\
\hline Health care workers' attitude was good or excellent & $25 / 33(76)$ & $69 / 72(96)$ \\
\hline Forensic exam was private & $12 / 34(36)$ & $70 / 72(97)$ \\
\hline Counselling was helpful & $16 / 26(62)$ & $64 / 65(99)$ \\
\hline \multicolumn{3}{|l|}{ Perceptions of police: } \\
\hline Police attitude was good or excellent & 18/34 (53) & $42 / 69(61)$ \\
\hline
\end{tabular}


Think that more than half of cases go to trial*

Confident own case will go to trial
$10 / 27(37)$

$8 / 22(36)$
$13 / 48(27)$

$11 / 50(22)$

PEP consists of provision of a full 28 day regimen of zidovudine and lamivudine to prevent HIV infection

${ }^{*}$ Among 144 rape cases presenting to the hospital between May 2004 and December 2005 in which a police report had been opened, only $2.8 \%$ of cases proceeded to trial.

\section{Table 2}

Patients' reports of completion of treatment and quality of care. Values are numbers (percentages) unless otherwise indicated

\section{Providing full PEP regimen on first visit}

Few rape survivors were willing or able to return for follow-up visits-a finding that is not confined to remote or resource poor settings.3 615161718 The intervention introduced a policy for prescribing a full PEP regimen to eligible patients on their initial hospital visit. Without further counselling visits, this could lead to poor adherence and a waste of expensive drugs—but patients who are unable to return to hospital and only complete a starter pack of PEP also receive incomplete treatment, so these pills too are effectively "wasted."

Patients given a full course of drugs on first visit were much more likely than those given a starter pack with follow-up appointments to have taken PEP for 28 days (71\% v 29\%). After the intervention, patients were more likely to report having received any HIV prophylaxis, to have received a full 28 day course on first visit, and to have completed the full regimen (table $2 \Uparrow$ ).

South African guidelines include a provision for dispensing the full PEP regimen on the initial visit for eligible patients unable to return because of economic or logistic reasons.10 Our findings support the feasibility and practicality of this recommendation. PEP guidelines highlight the importance of providing adequate counselling about drug treatment, alongside anti-emetics for control of nausea (a common side effect of the multiple drugs in the treatment package).2 10 In our study, the provision of antiemetics increased after the intervention, as did the proportion of patients who could report the correct dosing interval and purpose of PEP, suggesting that these elements may have contributed to the improvements in completion of PEP.

\section{Utilisation and quality of services}

The outpatient department's register indicated that use of the post-rape care service increased after the intervention, with the mean number of cases rising from 8 to 13 per month.9 Fewer patients were diverted to the police before being given treatment, and the proportion of patients who saw six or more service providers on their initial visit decreased from $86 \%$ to $54 \%$ (table $2 \mathbb{1}$ ). These improvements did not translate into a decrease in overall time spent at hospital, with similar proportions reporting they had stayed more than four hours. Patients' impressions of quality of care improved substantially, including their rating of providers' attitudes, the privacy of the forensic examination, and their perceptions of whether any counselling they had received had been helpful. However, patients' impressions of, or confidence in, the police and judicial system did not change.

\section{Role of nurses}

The role of nurses in care after sexual assault could be expanded substantially. At baseline, most care 
was delivered by doctors, with the nursing role confined primarily to obtaining the medical chart, taking vital signs, and helping the doctor. After the intervention, this role expanded to include documenting the rape history, providing acute trauma debriefing and an immediate dose of PEP drugs, and administering a pregnancy test. If certified to do so, the nurse would also offer HIV counselling and testing. Using a hospital protocol we developed during the intervention, nurses assembled the treatment package (drugs for sexually transmitted infections, emergency contraception, and HIV prophylaxis), provided counselling about drug treatment, and made psychosocial referrals.

A disappointing finding was the lack of impact on building nurses' capacity and willingness to perform the forensic examination. After the intervention, few nurses reported conducting an examination, and chart reviews indicated that $95 \%$ were conducted by a doctor. We probably underestimated the intensity of training required to develop proficiency and confidence in this area. Interviews showed that nurses were reluctant to learn about this aspect of post-rape management and were intimidated by the time needed for the examination and by the prospect of presenting evidence in court. Many thought that government policies were unclear about whether nurses' testimonies were admissible in court. These barriers have been reported in South Africa and elsewhere.19 202122 Although sexual assault nurse examiners have shown proficiency in the United States,23 24 in South Africa the training approaches and norms and standards for forensic nurses are still evolving. Further experience and research are needed.

Our findings show that it is possible to improve care after sexual assault, including giving HIV prophylaxis, within a public sector hospital, using existing staff and resources, and that with additional training, nurses can have an expanded role in this care. Although the study has important limitations, including the before and after design, it nonetheless highlights several practical lessons regarding management of sexual assault (box 2).

\section{Box 2: Emerging lessons for strengthening care after sexual assault in South Africa}

- Post-rape care can be effectively integrated into existing HIV and reproductive health services at the district hospital level in South Africa. Most diagnostic tests and treatments are available within hospitals, but they may be scattered across different departments and service providers, leading to obstacles and delays in providing care.

- A hospital rape management policy can establish an institutional framework for coordinating care. It should lay out the responsibilities of clerks, pharmacy staff, laboratory workers, counsellors, social workers, etc, as well as health care workers, as many of these providers impact directly on patient care. Such policies may also reduce the scope for individual providers to allow personal judgments and attitudes to shape their treatment of patients.

- A designated room for treating patients who have been sexually assaulted can reduce delays, centralise services, and increase privacy. Drugs and diagnostic tests can be stored and dispensed directly from this room, minimising delays and the need for additional providers.

- Treatment protocols can systematise care and make it easier for providers to follow national management guidelines. Such protocols are particularly important because of the lack of training received by many healthcare workers and their high turnover within health facilities.

- Most obstacles to providing HIV prophylaxis are institutional rather than patient driven. HIV infection is the patient's main health concern, and most come to hospital within 72 hours. Lack of 
HIV counselling and testing services at presentation may be a serious bottleneck. Because most patients present to hospital after hours, these services should be made available 24 hours a day.

- Because of conventional practices for dispensing drugs, post-exposure prophylaxis (PEP) may be the last step in the hospital visit. Providing an immediate dose of PEP can reduce the time interval to starting the regimen.

- In rural areas, few patients are able to return to hospital after initial presentation. Wherever possible, all diagnostic tests and treatment should be provided on the first visit, and the full 28 day course of PEP drugs should be dispensed on the first visit. Same-day provision of antiemetics and counselling about drug treatment are important for encouraging adherence.

- Nurses can play a much greater role in providing post-rape care, particularly in rural areas where there are few doctors. Further research and experience in training nurses to perform forensic examinations is needed to guide policy and practice.

- Working with other sectors is critical. Because most rape survivors present to the police first, cooperation with the police can lessen delays to treatment and provide an opportunity to strengthen medicolegal services. In the absence of rape crisis centers or other non-governmental organisations, ongoing counselling and support can be provided by strengthening referral systems to existing service providers, such as psychiatric nurses or social workers.

\section{Next steps}

Remaining challenges include sustaining the programme and sharing the lessons learnt. The estimated additional cost of delivering the intervention was 1406 Rand (about \$200) per case, including costs at the facility level (capital investment, training and development, salaries of the research nurse and coordinator of the sexual violence advisory committee) and patient level (drugs, tests, and referrals). When one-off development costs and initial salary costs were excluded, incremental costs decreased to 408 Rand (about \$58) per case.9 Protocols and monitoring and evaluation tools have been established within the hospital, and key statistics on HIV prophylaxis are now routinely reported to hospital management and district and provincial health managers. Lessons have been shared with the Department of Health, and more than 800 health managers and service providers have received training. Findings from the study have contributed to recent international policy and guidelines on postexposure HIV prophylaxis, 4 and the Refentse model is now informing efforts to improve care after sexual assault in eight countries within eastern and southern Africa.25 As initiatives to address sexual violence are expanded, it is critical that further research, particularly in developing countries, is used to inform programmes and policy.

\section{Notes}

Cite this as: BMJ 2009;338:b515

\section{Footnotes}

- We thank the members of the project advisory committee for their guidance throughout the study, the Department of Health and Social Welfare in Limpopo Province and Mpumalanga Province for their encouragement and support, the Western Cape Provincial Reference Group for Sexual 
Violence for their technical support in the training workshop and in developing intervention tools, and Carol Wiebe for invaluable contributions and support to the project team. We are indebted to the many dedicated service providers at the study site and the courageous women and children who, as survivors of sexual assault, shared their valuable insights with us.

- Contributors: JCK was the principal investigator of the study, led the drafting of this manuscript, and contributed to all aspects of the study. IA contributed to the overall design of the study and the initial conceptualisation of the intervention and provided support to the research team. LM contributed to the development of the intervention and conducted patient interviews. ND assisted with the design and conduct of key informant interviews and chart reviews and contributed to data management and analysis. TA was responsible for the statistical analysis. SJ led the design and conduct of the economic evaluation. EN contributed to the development and implementation of the intervention model. JC contributed to the study design and the conceptualisation of the intervention and provided support to the research team. CW contributed to the initial conceptualisation of the study and advised on most aspects of the study. All authors contributed to the drafting of this manuscript. JCK is guarantor.

- Funding: United States Agency for International Development (USAID) and the US President's Emergency Plan for AIDS Relief (PEPFAR) under the terms of Cooperative Agreement Number HRN-A-00-98-00012-00 and Subagreement numbers A104.25A and S107019A. The contents are the responsibility of the Population Council and do not necessarily reflect the views of USAID or the United States Government. The sponsors had no role in study design, data collection, analysis, interpretation or writing this report.

- Competing interests: None declared.

- Ethical approval: Ethical clearance for the study was obtained from the University of the Witwatersrand Human Research Ethics Committee (\#M060335), the London School of Hygiene and Tropical Medicine Ethics Committee (\#3036), and the Population Council IRB (Protocol No 352). All research participants provided informed consent. Permission to conduct the study was sought from hospital management, and feedback on study progress and results was delivered to a project advisory committee.

- Provenance and peer review: Not commissioned; externally peer reviewed.

\section{References}

1. World Health Organization. Sexual violence. In: World report on violence and health. Geneva: WHO, 2002. http://whqlibdoc.who.int/publications/2002/9241545615 chap6 eng.pdf

2. World Health Organization. Guidelines for medico-legal care for victims of sexual violence. Geneva: WHO, 2003. www.who.int/violence injury prevention/publications/violence/med leg guidelines/en/

3. Myles J, Hirozawa A, Katz M, Kimmerling R, Bamberger J. Postexposure prophylaxis for HIV after sexual assault. JAMA2000;284:1516-7.

4. International Labour Organization and World Health Organization. Occupational and non-occupational postexposure prophylaxis for HIV infection (HIV-PEP). Joint ILO/WHO technical meeting for the development of policy and guidelines: summary report. Geneva: ILO/WHO, 2005.

www.who.int/hiv/topics/prophylaxis/meeting/en/index.html

5. Kim J, Martin L, Denny L. Rape and HIV postexposure prophylaxis: addressing the dual epidemics in South 
Africa. Reprod Health Matters2003;11:101-12.

6. Roland ME. Postexposure prophylaxis after sexual exposure to HIV. Curr Opin Infect Dis2007;20:39-46.

7. Martin SL, Young SK, Billings DL, Bross CC. Health care-based interventions for women who have experienced sexual violence: a review of the literature. Trauma Violence Abuse2007;8:3-18.

8. Population Council Frontiers in Reproductive Health Program and School of Public Health, University of the Witwatersrand. Frontiers news: integrating post-rape care services into existing reproductive health programs in South Africa. Johannesburg: Population Council, 2005:6-7.

9. Kim JC, Askew I, Mokwena L, Dwane N, Abramsky T, Jan S, et al. Developing an integrated model for postrape care and HIV post-exposure prophylaxis in rural South Africa. 2007. www.popcouncil.org/pdfs/frontiers/FR FinalReports/SouthAfrica RADAR.pdf

10. South African National Department of Health. National management guidelines for sexual assault. Pretoria: South African National Department of Health, 2005.

11. World Health Organization. Putting women first: ethical and safety recommendations for research on domestic violence against women. Geneva: WHO, 2001. www.who.int/gender/violence/womenfirtseng.pdf

12. Ellsberg M, Heise L. Researching violence against women: a practical guide for researchers and activists. Washington, DC: World Health Organization, PATH, 2005. www.path.org/files/GBV rvaw complete.pdf

13. Gerberding JL. Management of occupational exposures to blood-borne viruses. N Engl J Med1995;332:44451.

14. Niu MT, Stein DS, Schnittman S. Primary HIV type-1 infection: review of pathogenesis and early treatment intervention in human and animal retrovirus infections. J Infect Dis1993;168:1490-501.

15. Wiebe ER, Comay SE, McGregor M, Ducceschi S. Offering HIV prophylaxis to people who have been sexually assaulted: 15 months' experience in a sexual assault service. CMAJ2000;162:641-5.

16. Linden JA, Oldeg P, Mehta SD, McCabe KK, LaBelle C. HIV post-exposure prophylaxis in sexual assault: current practice and patient adherence to treatment recommendations in a large urban teaching hospital. Acad Emerg Med2005;12:640-6.

17. Garcia MT, Fiqueiredo RM, Moretti ML, Resende MR, Bedoni AJ, Papaiordanou PMO. Postexposure prophylaxis after sexual assaults: a prospective cohort study. Sex Transm Dis2005;32:214-9.

18. Gibb AM, McManus T, Forster GE. Should we offer antibiotic prophylaxis post sexual assault? Int J STD AIDS2003;14:99-102.

19. Christofides NJ, Jewkes R, Webster N, Penn-Kekana L, Abrahams N, Martin LJ. "Other patients are really in need of medical attention"-the quality of health services for rape survivors in South Africa. Bull World Health Organ2005;83:495-502.

20. South African National Department of Health. Sexual assault policy. Pretoria, the Department, 2005.

21. Green WM, Panacek EA. Sexual assault forensic examinations in evolution. J Emerg Med2003;25:97-9.

22. Suffla S, Seedat M, Nascimento A. Evaluation of medico-legal services in Gauteng: implications for the development of best practices in the after-care of rape survivors. 2001. www.afrihealth.com/violence/medicobest.htm

23. Ledray LE, Simmelink K. Efficacy of SANE evidence collection: a Minnesota study. J Emerg Nurs1997;23:75-7.

24. Derhammer F, Lucente V, Reed JF, Young MJ. Using a SANE interdisciplinary approach to care of sexual assault victims. J Qual Improv2000;26:488-95.

25. Population Council. Sexual and gender-based violence in east and southern Africa, 2007. www.popcouncil.org/pdfs/AfricaSGBV Keylssues.pdf 
